\title{
Evaluation of Ipatasertib Interactions with Itraconazole and Coproporphyrin I and III in a Single Drug Interaction Study in Healthy Subjects $\$$
}

\author{
Rucha S. Sane, Kit Wun Kathy Cheung, Eunpi Cho, Bianca M. Liederer, Justin Hanover, \\ Vikram Malhi, Emile Plise, Susan Wong, and Luna Musib
}

Genentech, South San Francisco, California

Received Mar 19, 2021; accepted May 24, 2021

\begin{abstract}
Ipatasertib is a pan-AKT inhibitor in development for the treatment of cancer. Ipatasertib was metabolized by CYP3A4 to its major metabolite, M1 (G-037720), and was a P-gp substrate and OATP1B1/1B3 inhibitor in vitro. A phase I drug-drug interaction (DDI) study $(n=15)$ was conducted in healthy subjects to evaluate the effect of itraconazole (200-mg solution QD, 4 days), a strong CYP3A4 and P-gp inhibitor, on pharmacokinetics of ipatasertib (100-mg single dose). Itraconazole increased the $\mathrm{C}_{\max }$ and $\mathrm{AUC}_{0-\infty}$ of ipatasertib by 2.3- and 5.5-fold, respectively, increased the half-life by $53 \%$, and delayed the $t_{\max }$ by 1 hour. The $\mathrm{C}_{\max }$ and $\mathrm{AUC}_{0-72 \mathrm{~h}}$ of its metabolite M1 (G037720 ) reduced by $91 \%$ and $68 \%$, respectively. This study confirmed that CYP3A4 plays a major role in ipatasertib clearance. Furthermore, the interaction of ipatasertib with coproporphyrin (CP) I and CPIII, the two endogenous substrates of OATP1B1/1B3, was evaluated in this study. CPI and CPIII plasma levels were unchanged in the presence of ipatasertib, both at exposures of $100 \mathrm{mg}$ and at higher exposures in
\end{abstract}

combination with itraconazole. This indicated no in vivo inhibition of OATP1B1/1B3 by ipatasertib. Additionally, it was shown that $\mathrm{CPI}$ and $\mathrm{CPIII}$ were not P-gp substrates in vitro, and itraconazole had no effect on CPI and CPIII concentrations in vivo. The latter is an important finding because it will simplify interpretation of future DDI studies using CPI/CPIII as OATP1B1/ 1B3 biomarkers.

\section{SIGNIFICANCE STATEMENT}

This drug-drug interaction study in healthy volunteers demonstrated that CYP3A4 plays a major role in ipatasertib clearance, and that ipatasertib is not an organic anion transporting polypeptide 1B1/1B3 inhibitor. Furthermore, it was demonstrated that itraconazole, an inhibitor of CYP3A4 and several transporters, did not affect CPI/CPIII levels in vivo. This increases the understanding and application of these endogenous substrates as well as itraconazole in complex drug interaction studies.

\section{Introduction}

Ipatasertib (GDC-0068) is a novel, potent, highly selective small-molecule inhibitor of the three isoforms of serine/threonine kinase Akt (Akt1, Akt2, and Akt3) (Blake et al., 2012; Lin et al., 2013; Yan et al., 2013) or protein kinase B. It is

All studies were sponsored by Genentech Inc.

All authors were employees of Genentech, Inc., a member of the Roche Group, and holders of Roche Holding Ltd. stock when the work was performed.

This work was previously presented as a poster: R.S. Sane, E. Plise, V. Malhi, B. Chen, L. Musib (2019). Evaluation of Ipatasertib Interaction with a Strong CYP3A4 Inhibitor, Itraconazole and Endogenous Substrate of OATP1B1/1B3 Substrate Coproporphyrin I and III in One Drug-Drug Interaction Study. American College of Clinical Pharmacology 50th Annual Meeting; 2019 September 15-17; Chicago, IL.

https://dx.doi.org/10.1124/jpet.121.000620

S This article has supplemental material available at jpet.aspetjournals.org. being developed to be used in combination with chemotherapy, hormonal agents, or targeted agents for the treatment of breast and prostate cancers (Kim et al., 2017; de Bono et al., 2019).

In vitro assessments of drug metabolizing enzyme- and transporter-mediated pharmacokinetic drug-drug interactions (DDI) were conducted with ipatasertib, as patients with breast and prostate cancers may often require concomitant medications. Here, we present both the in vitro metabolism and transport studies as well as the clinical DDI study conducted to assess the clinical impact. In vitro, ipatasertib is primarily metabolized by cytochrome P450 (P450) 3A4. An N-dealkylated metabolite, M1 (G-037720), which circulates at concentrations of approximately $40 \%$ of the parent drug at steady state, is formed via CYP3A4 (Supplemental Fig. 1). Ipatasertib is also a substrate of the efflux transporter P-glycoprotein

ABBREVIATIONS: AUC, area under the curve; BCRP, breast cancer resistance protein; $\mathrm{CCK}-8$, $\left[{ }^{3} \mathrm{H}\right]$ cholecystokinine-8; $\mathrm{Cl}$, confidence interval; $\mathrm{CL} / \mathrm{F}$, oral clearance; $\mathrm{CP}$, coproporphyrin; $\mathrm{DDI}$, drug-drug interaction; $\mathrm{E}_{2} 17 \beta \mathrm{G}$, $\left[{ }^{3} \mathrm{H}\right]$ estradiol-17 $\beta$-glucuronide; HEK293, human embryonic kidney 293 cells; ITZ, itraconazole; LC-MS/MS, liquid chromatography-mass spectrometry; LLOQ, lower limit of quantitation; MDCK, Madin-Darby canine kidney; MDR1, Multidrug resistance protein 1; MRP, multidrug resistance-associated protein; MRAUC, metabolite/parent ratio based on AUC; MRCmax, metabolite/parent ratio based on maximal plasma concentration (Cmax); NMQ, N-methyl-quinidine; OATP, organic anion transporting polypeptide; P450, cytochrome P450; P-gp, P-glycoprotein; PK, pharmacokinetics; QD, daily; rCYP, recombinant cytochrome P450; $t_{1 / 2}$, half-life; TEAE, treatment-emergent adverse event; Imax, time to reach maximal concentration; 'Vz/F, apparent volume of distribution. 
(P-gp), but not a substrate of breast cancer resistance protein (BCRP) in vitro. Inducers of clearance pathways can reduce systemic exposures of the substrates of those pathways, and inhibitors can increase the systemic exposures (Venkatakrishnan et al., 2000; Prueksaritanont et al., 2017). Accordingly, enzalutamide, a potent CYP3A4 inducer, was coadministered with ipatasertib, it reduced exposure of ipatasertib by $\sim 50 \%$ (Isakoff et al., 2020). Similarly, coadministering inhibitors of CYP3A4 and P-gp may increase the systemic exposure of ipatasertib.

Furthermore, an in vitro transporter inhibition assay showed that ipatasertib was a weak inhibitor of organic anion transporting polypeptide (OATP) 1B1 and OATP1B3, with $\leq 25 \%$ increase in the exposure of a substrate predicted because of inhibition of either transporter. OATP1B1 and OATP1B3 play a crucial role in the hepatic uptake of both endogenous compounds and drugs such as statins (Liu et al., 2018). Regulatory authorities have provided detailed recommendations on evaluation of clinical DDI related to OATP1B1/1B3 (https://www.fda.gov/media/108130/download; https://www.ema.europa.eu/en/documents/scientific-guideline/ guideline-investigation-drug-interactions-revision-1_en.pdf; https://www.pmda.go.jp/files/000228122.pdf) if the expected increase in exposure of substrates is $>4$ and $10 \%$ ratio of area under the plasma concentration time curve (AUCR) cut off values of 1.04 for European Medicines Agency (EMA) and 1.1 for the Food and Drug Administration (FDA) and Pharmaceuticals and Medical Devices Agency (PMDA). Recent research has identified coproporphyrin (CP) I and CPIII, the porphyrin metabolites from heme synthesis, as promising endogenous biomarkers for OATP activity (Lai et al., 2016) and can aid in assessing the effect of OATP1B1/1B3 inhibition in vivo. Evaluation of CPI and CPIII as endogenous biomarkers can assist in further understanding the need for a clinical DDI study.

To investigate the in vivo effect of CYP3A and P-gp inhibition on ipatasertib, a dedicated DDI study with itraconazole (ITZ), a strong dual CYP3A4 and P-gp inhibitor, was conducted. Within this same study, the effect of ipatasertib on the plasma levels of CPI and CPIII were assessed to evaluate the in vivo significance of in vitro OATP1B1/OATP1B3 inhibition observed with ipatasertib. A DDI between ITZ and ipatasertib was expected to increase ipatasertib exposure. Therefore, to evaluate the effect of increased ipatasertib exposure on OATP activity, CPI and CPIII exposures were measured when ipatasertib was coadministered with ITZ. Given that ITZ is a P-gp inhibitor, an in vitro P-gp vesicular transport experiment was performed with CPI and CPIII to rule out any role of P-gp in CPI and CPIII disposition. Additionally, the in vivo effect of ITZ on CPI and CPIII exposure was investigated in vivo.

\section{Materials and Methods}

\section{In Vitro CYP3A4 and P-gp Substrate Assay for Ipatasertib}

CYP3A4 Substrate Assay. To identify the P450 isoforms responsible for the metabolism of ipatasertib, pooled human liver microsomes (Corning, Tewksbury, MA) were incubated with ipatasertib in the presence or absence of chemical inhibitors of specific P450 isoforms. Selective P450 inhibitors (furafylline for CYP1A2, trancylcypromine for CYP2A6, ticlopidine for CYP2B6, quercetin for CYP2C8, sulfaphnazole for CYP2C9, ticlopidine for CYP2C19, quinidine for CYP2D6, ketoconazole for CYP3A4/5) or P450 inactivators (troleandomycin and 1-aminobenzotriazole) were preincubated with human liver microsomes and NADPH for 5 or 15 minutes at $37^{\circ} \mathrm{C}$ with $\mathrm{NADPH}(1 \mathrm{mM})$. Reactions were initiated with the addition of ipatasertib. Samples were collected from each HLM reaction at the 0- and 60-minute timepoints, centrifuged, and the supernatant was analyzed for ipatasertib and M1 (G-037720) by liquid chromatography-mass spectrometry (LC-MS/MS).

Recombinant Cytochrome P450 Assay. Various rCYP isoforms (rCYP1A2, rCYP2A6, rCYP2B6, rCYP2C8, rCYP2C9, rCYP2C18, rCYP2C19, rCYP2D6, rCYP2E1, rCYP3A4, and rCYP3A5) were used as a qualitative assessment for the formation of the metabolites. rCYP $\left(40 \mathrm{pmol} / \mathrm{mL}\right.$ ) and NADPH were preincubated for 5 minutes at $37^{\circ} \mathrm{C}$. The reaction was initiated with the addition of ipatasertib $(1 \mu \mathrm{M})$ and quenched after 60 minutes. Samples were centrifuged, and the supernatant was analyzed by LC-MS/MS. Formation of M1 (G-037720) was monitored after incubations of ipatasertib with various rCYP isoforms.

P-gp and BCRP Substrate Assay. To assess whether ipatasertib was a substrate of P-gp and BCRP, Madin-Darby canine kidney (MDCK) II transfected with human MDR1 gene (MDCKII-MDR1) or human BCRP gene (MDCKII-BCRP) were used. Monolayer assay was performed at three concentrations of ipatasertib $(0.2,2$, and $60 \mu \mathrm{M}$, representing a range bracketing expected maximal plasma concentration of $\sim 1.56 \mu \mathrm{M})$. The highest concentration represented the maximum tolerated concentration in the assay. Briefly, assay buffer containing ipatasertib in the presence and absence of PSC833 (P-gp inhibitor) (Solvo Biotechnology, Szeged, Hungary) or Ko134 (BCRP inhibitor) was added to the appropriate apical or basolateral chamber and incubated at $37^{\circ} \mathrm{C}$. Digoxin or prazosin (Sigma-Aldrich, St Louis, MO) efflux ratio was determined as a positive control for MDR1 or BCRP function, with and without PSC833. Ipatasertib or control samples were taken from the receiver chambers at 0 and 120 minutes to determine the amount that permeated through the monolayer. Ipatasertib samples were analyzed by LC-MS/MS, whereas $\left[{ }^{3} \mathrm{H}\right]$ digoxin and $\left[{ }^{3} \mathrm{H}\right]$ prazosin (Perkin Elmer, Waltham, MA) were analyzed by liquid scintillation counting.

\section{In Vitro Inhibitory Assay of Ipatasertib on Uptake Activity of OATP1B1/3}

The effect of ipatasertib on OATP1B1 and OATP1B3-mediated transport was determined using intracellular accumulation of the probe substrates $\left[{ }^{3} \mathrm{H}\right]$ estradiol- $17 \beta$-glucuronide $\left(\mathrm{E}_{2} 17 \beta \mathrm{G}\right)$ and $\left[{ }^{3} \mathrm{H}\right]$ cholecystokinine-8 (CCK-8), respectively, in HEK293K cells overexpressing either OATP1B1 or OATP1B3 in the presence of ipatasertib as previously described (Sane et al., 2020).

Cell Culture. HEK293 cells were transfected with vectors containing cDNA for OATP1B1, OATP1B3, or vectors only by Solvo Biotechnology, Inc (Szeged, Hungary). HEK293 cells overexpressing OATP1B1, OATP1B3, and empty vector were cultured in Dulbecco's modified Eagle's medium supplemented with fetal bovine serum $(8.9 \%)$, antibiotic/antimycotic $(0.89 \%)$, and L-glutamate $(1.79 \mathrm{mM})$ in a humidified culture chamber $\left(37 \pm 1^{\circ} \mathrm{C}, 95 \pm 5 \%\right.$ relative humidity, and $5 \pm 1 \% \mathrm{CO}_{2}$ ). The medium was replaced every 2 to 3 days, and the cells were passaged when they became confluent.

OATP Inhibition Assay. Ipatasertib, $\mathrm{E}_{2} 17 \beta \mathrm{G}$ (Sigma-Aldrich, St Louis, MO), and CCK-8 (Sigma-Aldrich, St Louis, MO) were prepared in DMSO spiked into incubation medium at $0.1 \%$ DMSO. The cells were plated onto standard 96 -well tissue culture plates in cell culture medium 1 to 3 days prior to the experiment. OATP1B- and OATP1B3expressing and control cells were incubated with butyric acid for 24 hours prior to the experiment to inhibit suppression of the transporter. Incubations of HEK293 cells were carried out in incubation medium Henseleit-Krebs (HK) buffer. Prior to the experiment, the cells were rinsed once with $1 \mathrm{~mL}$ of incubation medium. Cells were then preincubated with $0.3 \mathrm{~mL}$ of incubation medium containing ipatasertib or solvent control. After 15 minutes, the medium was replaced with incubation medium containing $50 \mathrm{nM}$ of $\mathrm{E}_{2} 17 \beta \mathrm{G}$ (OATP1B1) or CCK-8 (OATP1B3) and ipatasertib or solvent control. After 3 
(OATP1B1) or 10 minutes (OATP1B3), incubation medium was removed and the cells were rinsed once with $1 \mathrm{~mL}$ of ice-cold phosphate-buffered saline containing $0.2 \%$ bovine serum albumin and twice with ice-cold phosphate-buffered saline. The cells were then lysed with $0.5 \mathrm{~mL}$ of $0.1 \mathrm{M}$ sodium hydrozide. Aliquots of the cell lysate were transferred to a 96-well plate, diluted with scintillation fluid, and analyzed on a MicroBeta2 liquid scintillation counter (Perkin Elmer, Waltham, MA). The amount of protein in each incubation was determined by bicinchoninic acid analysis.

\section{CPI and CPIII P-gp Vesicular Transport Experiment}

CPI and CPIII were tested at 2.5 and $10 \mathrm{nM}$ using the Solvo PREDIZEV MDR1 Vesicular Kit in accordance with the protocol with minor modifications (Solvo Biotechnology, Szeged, Hungary). Briefly, CPI, CPIII, and $N$-methyl-quinidine (NMQ) (positive control, HerediSzabo et al., 2013) were added to the plate containing vesicles and allowed to equilibrate for 15 minutes at $37^{\circ} \mathrm{C}$. The reaction was initiated by adding the ATP and AMP solutions. The plate was gently mixed on a plate shaker and placed in a $37^{\circ} \mathrm{C}$ incubator. At 5,10 , and 20 minutes, $200 \mathrm{uL}$ of ice-cold wash buffer was added to vesicle samples to quench the reaction. All solution was transferred to a prewetted filter plate and vacuum ( $\sim 6 \mathrm{psi})$ was applied until all liquid was removed. Wells were washed 5 times with $200 \mathrm{uL}$ of wash buffer. Methanol/water (70:30, V/V) containing $100 \mathrm{nM}$ propranolol as internal standard was added to lyse the vesicles. CPI and CPIII samples were transferred to a clear bottom plate and were read on a SpectaMax i3 plate reader using an excitation wavelength of $390 \mathrm{~nm}$ and an emission wavelength of 621 . NMQ samples were analyzed based on Solvo's protocol. Samples were injected onto a Phenomenex XB C18 column $(50 \times 2 \mathrm{~mm})$ using water with $0.1 \%$ formic acid (mobile phase A) or acetonitrile with $0.1 \%$ formic acid (mobile phase B). Elution was carried out under gradient conditions (acetonitrile with $0.1 \%$ formic acid). Samples were quantified using electrospray ionization (ESI) positive mode (+) and MRM mode on a Sciex 5500 Qtrap using the MRM mode with the transition $339 \rightarrow 160$.

\section{Clinical DDI Study}

A phase 1, single-center, open-label, two-period, fixed-sequence, DDI study in healthy subjects was conducted to evaluate the effect of ITZ on the pharmacokinetics (PK) of ipatasertib and its primary metabolite M1 (G-037720). The protocol, informed consent forms, and any other information given to the subjects were submitted to the Institutional Review Board for review and approved before the study was initiated. Written informed consent for the study was obtained from all subjects before protocol-specific procedures were carried out.

Study Population and Design. Healthy males and females (nonpregnant or nonlactating, and must be postmenopausal or surgically sterile or agree to use contraception from 10 days prior to study conduct until 30 days after the last dose of the study drug) who aged 18-55 years were screened to assess their eligibility to participate in the study within 26 days prior to study entry. Subjects were not allowed any concomitant medications from 14 days prior to the study. Foods known to affect CYP3A4, such as grapefruit juice, were also prohibited. Eligible subjects were admitted to the study site on the day prior to the first ipatasertib dosing (day -1) to collect baseline data for CPI and CPIII. All enrolled subjects were administered single oral doses of ipatasertib $(100 \mathrm{mg}$ ) on 2 study days (with or without coadministration of ITZ). Dosing details were as follows (Fig. 1):

On day 1 of period 1, subjects received a single oral 100-mg tablet of ipatasertib after at least an 8-hour fast, followed by a washout period of approximately 14 days between dosing on day 1 (period 1) and day 15 (period 2). On day 15 through day 23 (period 2), subjects received oral $200-\mathrm{mg}$ ITZ dose QD (20 mL x $10 \mathrm{mg} / \mathrm{mL}$ solution; QD), and on day 19 (period 2), ITZ was coadministered with a single oral 100-mg ipatasertib dose after at least an 8-hour fast. Doses (ipatasertib and ITZ) were followed by at least a 4-hour postdose fast. For each dose, except as part of dose administration, subjects were restricted from consumption of water for 1 hour prior to dose and for 2 hours postdose; at other times during the study, subjects consumed water ad libitum.

To assess the safety and tolerability of ipatasertib and ITZ, physical examinations, 12-lead electrocardiograms, vital signs, clinical laboratory evaluations, and recording of adverse events were performed at screening, at specified times during the study, and at follow-up or study completion and/or at early termination.

Plasma Samples Collection. Serial blood samples were collected for the determination of ipatasertib and M1 (G-037720) concentrations on days 1 to 7 (period $1 ; 0,0.167,0.5,1,2,3,4,6,8,12,24,36,48,72$, 96,120 , and 144 hours) and on days 19 through 27 (period 2; 0, 0.167, $0.5,1,2,3,4,6,8,12,24,36,48,72,96,120,144,168$, and 192 hours). Blood samples were collected for the determination of ITZ and its metabolite 1-hydroxyitraconazole concentrations on day -1 (period 1 ; baseline) and on days 18 and 19 (period 2; postdose). Serial blood samples were also collected for the determination of OATP biomarker CPI and CPIII concentrations over 24 hours prior to first ipatasertib dosing starting on day -1 through 24 hours postdose on day 2 (period 1) and on days 18 and 19 (period 2) prior to the second ipatasertib dose coadministered with ITZ.

Bioanalysis of Plasma Samples, PK, and Statistical Analyses. Plasma concentrations of ipatasertib, M1 (G-037720), ITZ, and 1hydroxyitraconazole were determined using a validated assay using supported-liquid extraction and high-performance liquid chromatography coupled with tandem mass spectrometric detection. CPI and CPIII plasma concentrations were determined by a qualified bioanalysis assay using liquid-liquid extraction and high-performance liquid

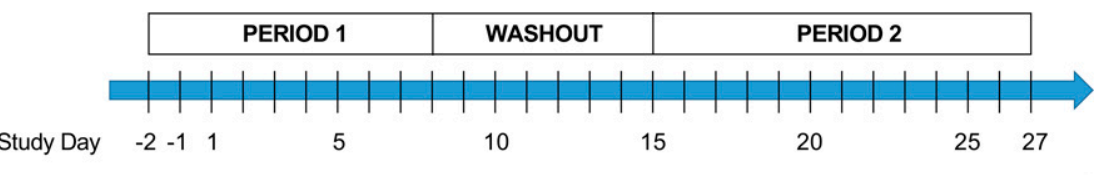

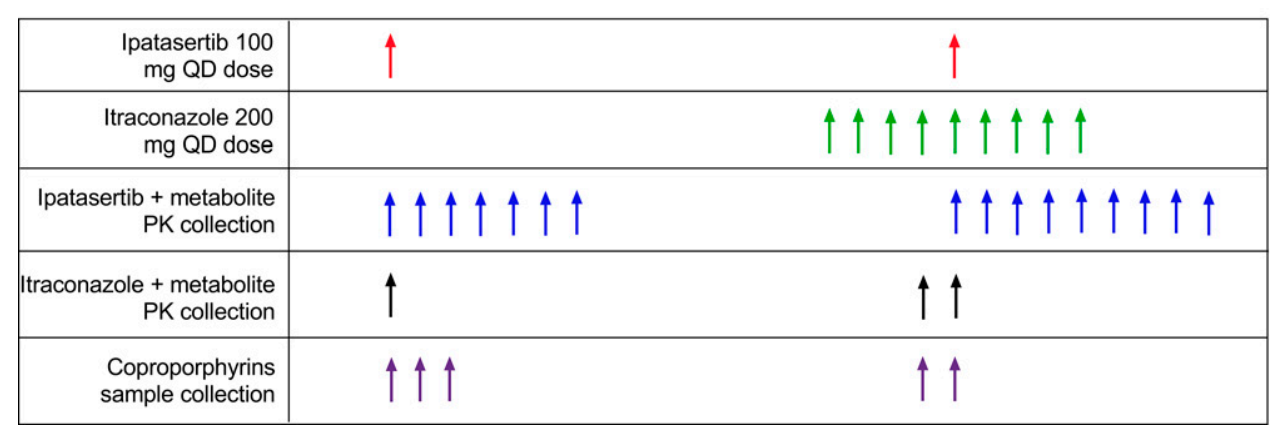

Fig. 1. Schematic of study design. 
chromatography-tandem mass spectrometry with multiple reaction monitoring and electrospray ionization in the positive mode by Covance Laboratories, Inc (Madison WI), (Liu et al., 2018). The LC-MS/MS analysis was carried out with an ACE C18 Column $(3 \mu \mathrm{m}, 50 \times 3 \mathrm{~mm})$ on a Shimadzu High Performance Liquid Chromatography system coupled with a Sciex API 5000 mass spectrometer. Chromatographic separation was performed under gradient conditions with a mobile phase composed of $10 \mathrm{mM}$ ammonium formate (mobile phase A) and methanol (mobile phase B). Ipatasertib, M1 (G-037720), ITZ, 1hydroxyitraconazole, and CPI/CPIII were monitored by $\mathrm{m} / \mathrm{z} 460.2 \rightarrow$ $389.2 ; 416.2 \rightarrow 217.2 ; 707.4 \rightarrow 392.3 ; 721.4 \rightarrow 408.3$; and $655.3 \rightarrow 596.3$, respectively. Assay employed deuterated internal standards for ipatasertib, M1, ITZ, and 1-hydroxy itraconazole or stable labeled internal standards for coproporphyrin. The LLOQ for ipatasertib and M1 was $0.463 \mathrm{ng} / \mathrm{mL}$, LLOQ for ITZ and 1-hydroxy itraconazole was $5 \mathrm{ng} / \mathrm{mL}$, LLOQ for CPI was $50 \mathrm{ng} / \mathrm{mL}$, and LLOQ for CPIII was $20 \mathrm{ng} / \mathrm{mL}$.

The following PK parameters were derived from plasma concentrations of ipatasertib and M1 (G-037720) using the noncompartmental methods: $\mathrm{C}_{\max }, \mathrm{t}_{\max }, \mathrm{AUC}_{0-\mathrm{tz}}, \mathrm{AUC}_{0-\infty}, t_{1 / 2}, \mathrm{CL} / \mathrm{F}$ (ipatasertib only), $\mathrm{V}_{\mathrm{Z}} / \mathrm{F}$ (ipatasertib only), metabolite ratio based on metabolite and parent $\mathrm{C}_{\max }$ and AUC. For CPI and CPIII analysis, $\mathrm{C}_{\max }, \mathrm{t}_{\max }$, and $\mathrm{AUC}_{0-\mathrm{tz}}$ were determined. PK calculations were performed using commercial software Phoenix WinNonlin (Certara Inc., version 6.4 or higher).

For the primary analysis of DDI with ITZ, the effect of ITZ on ipatasertib and M1 (G-037720) PK was assessed. Natural log-transformed $\mathrm{C}_{\text {max }}, \mathrm{AUC}_{0-\mathrm{t}}$, and $\mathrm{AUC}_{0-\infty}$ values for ipatasertib were compared between period 1 (reference; ipatasertib alone) and period 2 (test; ipatasertib plus ITZ) using a mixed effects model with treatment as a fixed effect and subject as a random effect. A point estimate and the corresponding 90\% confidence intervals (CIs) for the difference between least squares mean of the log-transformed $\mathrm{C}_{\max }, \mathrm{AUC}_{0-\mathrm{t}}$, and $\mathrm{AUC}_{0-\infty}$ with and without coadministration of ITZ were calculated and used to determine the presence of a DDI. For the analysis related to OATP1B1/1B3 inhibition, $\mathrm{AUC}_{0-24}$ and $\mathrm{C}_{\max }$ for CPI and CPIII were also compared in a similar way. All calculations were performed using SAS version 9.3 or greater.

\section{Results}

\section{In Vitro Metabolism of Ipatasertib to M1 (G-037720)}

HLM in the presence of selective chemical inhibitors and human rCYP isoforms were used to identify the major human P450 isoforms responsible for the metabolism of ipatasertib and formation of M1 (G-037720). Metabolism of ipatasertib in HLM was inhibited by CYP3A selective inhibitor, ketoconazole, as well as troleandomycin by $>94 \%$, but not other CYP450 inhibitors. These selective CYP3A inhibitors inhibited the formation of M1 (G-037720), suggesting CYP3A was the major contributor to the metabolism of ipatasertib. Incubation of ipatasertib with several recombinant $\mathrm{P} 450$ isoforms (rCYP1A2, rCYP2B6, rCYP2C8, rCYP2C9, rCYP2C19, rCYP2D6, rCYP3A4, rCYP3A5) confirmed that CYP3A4 was the primary P450 responsible for the formation of M1 (G037720). M1 itself had very low turnover in vitro.

Reaction phenotyping results are reported in Supplemental Table 1 in the supplemental file.

\section{In Vitro P-gp Substrate Assay}

Ipatasertib incubated at $0.2,2$, and $60 \mu \mathrm{M}$ in MDCKIIMDR1 monolayers resulted in efflux ratios of $1.41,6.14$, and 12.64 , respectively, which reduced to $1.20,1.78$, and 1.73 in the presence of the P-gp inhibitor, PSC833. These results suggested that ipatasertib was a substrate of P-gp. Results are shown in Supplemental Fig. 2 in the supplemental file.

\section{In Vitro BCRP Substrate Assay}

Ipatasertib incubated at $0.2,2$, and $60 \mu \mathrm{M}$ in MDCKII$\mathrm{BCRP}$ monolayers resulted in efflux ratios of $>6$. The presence of BCRP inhibitor Ko134 did not significantly reduce the efflux, however, the P-gp inhibitor PSC833 reduced the efflux ratio to 1 . This suggests the efflux observed was due to endogenous canine Mdr1 present in the cell line and not due to BCRP. Results are shown in Supplemental Fig. 3 in the supplemental file.

\section{In Vitro Inhibitory Assay of Ipatasertib on Uptake Activity of OATP1B1/3}

Ipatasertib inhibited the OATP1B1- and OATP1B3-mediated transport of $\mathrm{E}_{2} 17 \beta \mathrm{G}$ and cholecystokinin-8 (CCK-8), respectively, in a dose-dependent manner (Fig. 2). The calculated $\mathrm{IC}_{50}$ values were $141.5 \mu \mathrm{M}$ and $191.1 \mu \mathrm{M}$ for OATP1B1 and OATP1B3, respectively. Rifampicin $(50 \mu \mathrm{M})$ was used as a positive control, and at this concentration it completely inhibited OATP1B1 and 1B3 mediated uptake.

CPI and CPIII P-gp Vesicular Transport Experiment. The mean uptake of CPI and CPIII in MDR1 membrane vesicles in the presence of AMP and ATP were determined and demonstrated no active uptake (Fig. 3). Two concentrations of CPI and CPIII were tested (2.5 nM and 10 $\mathrm{nM}$ ), and no significant differences in the transport of CPI and CPIII at both tested concentrations were observed in the presence and absence of ATP. The positive control, NMQ, demonstrated marked uptake.

\section{Clinical DDI Study}

Demographics and Other Baseline Characteristics of Subjects. Fifteen subjects were enrolled in and completed the study. The median (range) age of all enrolled subjects was 36 years (22 to 52 years). Majority of the subjects were female $(66.7 \%)$, white $(53.3 \%)$, and Hispanic or Latino $(60.0 \%)$. The median (range) body mass index for enrolled subjects was $24.88 \mathrm{~kg} / \mathrm{m}^{2}$ (20.69 to $\left.30.24 \mathrm{~kg} / \mathrm{m}^{2}\right)$.

Ipatasertib Pharmacokinetics in the Absence and Presence of Itraconazole. Ipatasertib $\mathrm{C}_{\max }$ and AUC both showed a marked increase in presence of ITZ (Fig. 4; Table 1). The $t_{\max }$ appeared slightly delayed, and the arithmetic mean $t_{1 / 2}$ was 23 hours longer (approximately $53 \%$ increase) when ipatasertib was coadministered with ITZ versus alone. Statistical analysis to evaluate the effect of ITZ on the PK of ipatasertib yielded geometric least-square (LS) means ratios $(90 \%$ CIs) of approximately 545\% (496\% to 598\%) and 226\% (183\% to $280 \%$ ) for $\mathrm{AUC}_{0-\infty}$ and $\mathrm{C}_{\max }$, respectively (Table 2). That is, $\mathrm{AUC}_{0-\infty}$ and $\mathrm{C}_{\max }$ of ipatasertib increased by 5.45 -fold and 2.26-fold, respectively.

After single-dose oral administration of ipatasertib $100 \mathrm{mg}$ alone, absorption of ipatasertib was rapid, with detectable plasma concentrations for most subjects observed at the first sampling timepoint (Fig. 4; Table 1). After oral dosing of ipatasertib $100 \mathrm{mg}$ coadministered with ITZ $200 \mathrm{mg}$, first detectable concentrations of ipatasertib were not observed until approximately 30 minutes postdose. Geometric mean CL/F and $\mathrm{V}_{\mathrm{z}} / \mathrm{F}$ values decreased by approximately $82 \%$ and $72 \%$, respectively, after ipatasertib coadministered with ITZ versus ipatasertib alone.

Metabolite M1 (G-037720) Pharmacokinetics. Similar to the parent, M1 (G-037720) PK altered when ipatasertib 

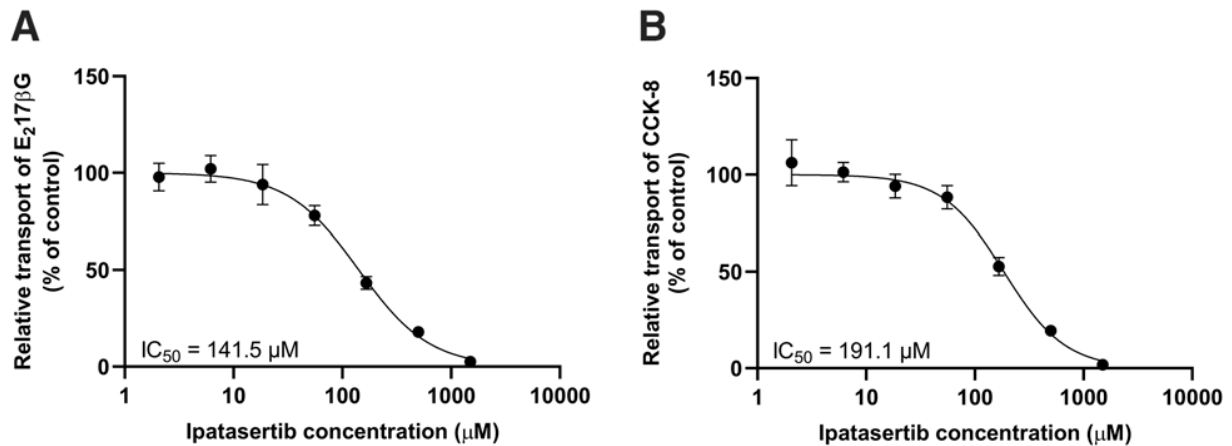

Fig. 2. A. Inhibition of OATP1B1-mediated E217 $\beta \mathrm{G}(1 \mu \mathrm{M})$ and B. OATP1B3-mediated CCK-8 $(0.1 \mu \mathrm{M})$ transport by ipatasertib was assessed in HEK293K cells stable transfected with respective transporters. Cells were dosed with ipatasertib and vehicle (1\% DMSO). The experiment was conducted for 2 minutes at $37^{\circ} \mathrm{C}$ for both transporters. Data are expressed as a mean $(n=3) \pm$ S.D. from a single experiment.

was administered with ITZ, with $\mathrm{C}_{\max }$ and AUC both showing marked decrease in presence of ITZ (Fig. 4; Table 3). Arithmetic mean $t_{1 / 2}$ of M1 (G-037720) were 36.0 hours and 47.6 hours when ipatasertib was coadministered with ITZ versus ipatasertib alone, respectively. Statistical analysis to evaluate the effect of ITZ on the PK of M1 (G-037720) yielded geometric least-square (LS) means for $\mathrm{AUC}_{0-72}$ and $\mathrm{C}_{\max }$ of approximately $68 \%$ and $91 \%$, respectively (Table 2 ). Coadministration of ipatasertib with ITZ decreased the geometric mean metabolic ratios $\left(\mathrm{MR}_{\mathrm{Cmax}}, \mathrm{MR}_{\mathrm{AUC0}-\infty}\right.$ and $\left.\mathrm{MR}_{\mathrm{AUC0}-72}\right)$ by approximately 25-, 12-, and 15-fold, respectively (Table 3 ).

After single-dose oral administration of ipatasertib $100 \mathrm{mg}$ alone, M1 (G-037720) detectable plasma concentrations appeared within 30 minutes for most subjects (Fig. 4; Table 3). Whereas, after oral dosing of ipatasertib $100 \mathrm{mg}$ coadministered with ITZ $200 \mathrm{mg}$, first detectable concentrations of M1 (G-037720) were not observed for most subjects until approximately 2 hours postdose. Median $t_{\text {max }}$ appeared delayed by about 6 hours when ipatasertib was coadministered with ITZ compared with ipatasertib alone (Table 3).

Pharmacokinetic Results for Itraconazole and Metabolite 1-Hydroxyitraconazole. Plasma concentrations of ITZ and its primary metabolite (1-hydroxyitraconazole) were measured predose and postdose on day $18\left(4^{\text {th }}\right.$ consecutive day of ITZ dosing) over a 24-hour period. After administration of ITZ, geometric mean (geometric CV\%) $\mathrm{C}_{\max }$ and median (min, max) $t_{\text {max }}$ for ITZ were $1640 \mathrm{ng} / \mathrm{mL}(17.5 \%)$ and $3.00(2.00,4.03)$ hours, respectively. In general, geometric mean ITZ trough (predose) concentrations on days 18 and 19

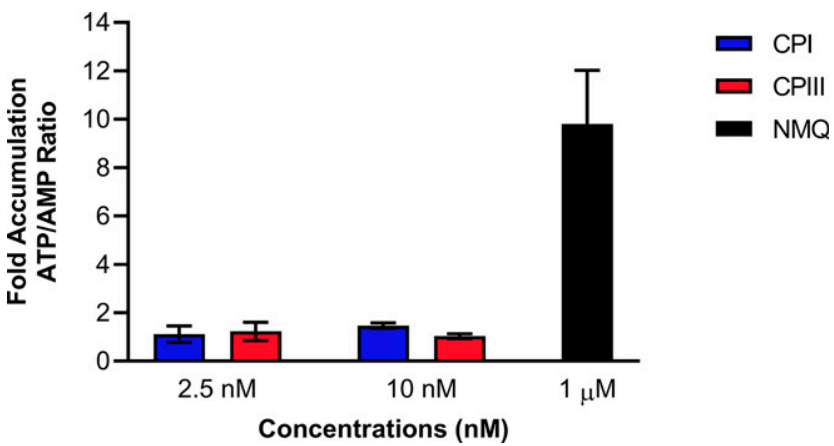

Fig. 3. Mean uptake of CPI and III in MDR1 membrane vesicles in the presence of AMP and ATP with total vesicle protein of $50 \mu \mathrm{g} / \mathrm{well}$. Ipatasertib was preinubated with vesicles for 10 minutes before the addition of ATP/AMP to start the reaction. Data are expressed as a mean $(n=3) \pm$ S.D. from a single experiment. were similar (313 and $383 \mathrm{ng} / \mathrm{mL}$, respectively). For 1-hydroxyitraconazole, geometric mean (geometric $\mathrm{CV} \%$ ) $\mathrm{C}_{\max }$ and median (min, max) $\mathrm{t}_{\max }$ were $1280 \mathrm{ng} / \mathrm{mL}(15.6 \%)$ and 4.00 $(3.00,5.00)$ hours, respectively.

CPI and CPIII Plasma Levels. Ipatasertib, at the exposures achieved in this study with or without ITZ, did not cause any changes in CPI and CPIII plasma levels (Fig. 5; Tables 4 and 5). ITZ administration also did not alter the CPI and CPIII plasma levels (Fig. 5; Table 4).

Summary of Adverse Events. Overall, both ipatasertib and ITZ were well tolerated when given alone or in combination to the healthy male and female subjects in this study. All
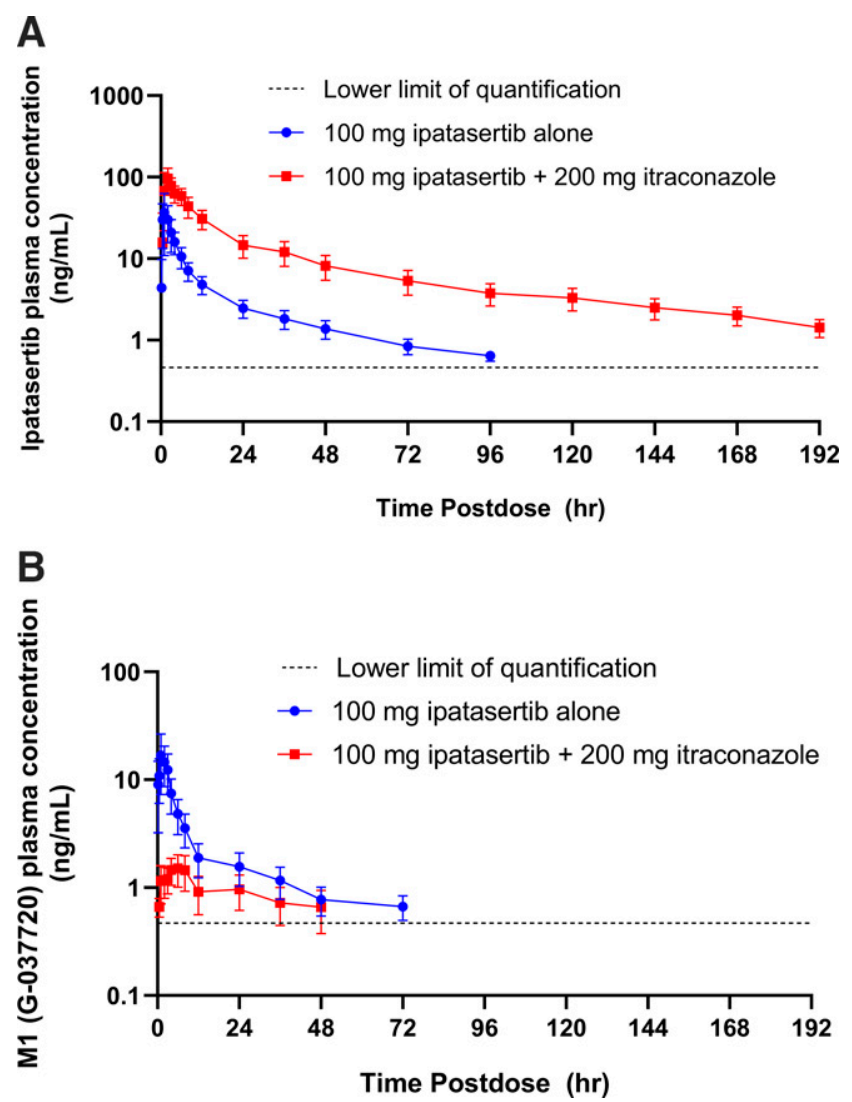

Fig. 4. Arithmetic mean (+S.D.) plasma concentration-time profiles of ipatasertib (A) and the major metabolite M1 (G-037720) (B) after singledose oral administration of ipatasertib $100 \mathrm{mg}$ alone or coadministered with itraconazole $200 \mathrm{mg}$ in healthy subjects $(N=15) . \mathrm{hr}=$ hour. 
TABLE 1

Summary of PK parameters of ipatasertib after single oral dose of ipatasertib 100 mg alone or coadministered with itraconazole 200 mg

All values are reported as geometric mean (geo\% CV); $\mathrm{t}_{\max }$ is reported as median (range); $t_{1 / 2}$ is reported as mean (S.D.).

\begin{tabular}{|c|c|c|}
\hline Ipatasertib PK Parameters & Ipatasertib (100 mg) & Ipatasertib $(100 \mathrm{mg})+$ Itraconazole $(200 \mathrm{mg})$ \\
\hline & $N=15$ & $N=15$ \\
\hline $\mathrm{AUC}_{0-\infty}(\mathrm{ng} * \mathrm{~h} / \mathrm{mL})$ & $327(26.4)$ & $1780(22.6)$ \\
\hline AUC0-72 (ng*h/mL) & $271(25.5)$ & $1300(22.9)$ \\
\hline $\mathrm{C}_{\max }(\mathrm{ng} / \mathrm{mL})$ & $44.9(35.9)$ & $102(34.1)$ \\
\hline$t_{\max }(h)$ & $1.07(0.50,3.03)$ & $2.05(1.00,6.00)$ \\
\hline$t_{1 / 2}(\mathrm{~h})$ & $44.0(8.52)$ & $67.2(9.20)$ \\
\hline $\mathrm{CL} / \mathrm{F}(\mathrm{L} / \mathrm{h})$ & $306(26.4)$ & $56.2(22.6)$ \\
\hline $\mathrm{Vz} / \mathrm{F}(\mathrm{L})$ & $19100(20.0)$ & $5400(33.9)$ \\
\hline
\end{tabular}

treatment-emergent adverse events (TEAEs) were assessed by the investigator as mild (grade 1) in intensity and resolved by the end of the study. Three subjects had TEAEs that were assessed by the investigator as related to ipatasertib; these TEAEs considered related to ipatasertib were nausea. Four subjects had TEAEs that were assessed by the investigator as related to ITZ; these TEAEs considered related to ITZ included nausea (three subjects) and diarrhea, dysgeusia, headache, and disorientation (one subject each). No clinically significant changes from baseline or findings were noted from clinical laboratory evaluations, vital signs measurements, or 12-lead ECGs for this study.

\section{Discussion}

Itraconazole had a marked impact on PK of ipatasertib: ipatasertib exposure $\left(\mathrm{AUC}_{0-\infty}\right)$ increased by 5.45 -fold, the mean $t_{1 / 2}$ increased by $53 \%$, and the CL/F decreased by $82 \%$ in the presence of itraconazole. Ipatasertib is a substrate of both CYP3A4 and P-gp in vitro, and the results are consistent with the inhibition of CYP3A4- and P-gp-mediated clearance by itraconazole. The inhibitory effect of itraconazole on CYP3A4 versus P-gp cannot be differentiated easily; however, the impact of itraconazole on M1 (G-037720), the major metabolite of ipatasertib, can provide additional insights. The $\mathrm{C}_{\max }$ and the $\mathrm{AUC}_{0-72}$ of $\mathrm{M} 1$ (G-037720), a metabolite formed primarily via CYP3A4, decreased by $91 \%$ and $68 \%$, respectively, suggesting CYP3A4 has a major role in the elimination of ipatasertib and may be the main mechanism underlying the DDI. This also lends additional support to the observation that M1 follows formation rate limited kinetics apparent from the similar half-lives of ipataseritb and M1 when ipatasertib is administered alone.

TABLE 2

Statistical analysis of ipatasertib and M1 (G-037720) PK parameters after single oral dose of ipatasertib $100 \mathrm{mg}$ alone or when coadministered with itraconazole $200 \mathrm{mg}$

Geometric mean ratio (GMR) is ratio of the least squares means from ANOVA for natural log-transformed AUCs or $\mathrm{C}_{\max }$ (expressed as a percent), natural log was transformed back to the linear scale; Test/Reference where test $=$ ipatasertib + itraconazole and reference $=$ ipatasertib alone.

\begin{tabular}{lcc}
\hline Analyte & Parameter & GMR $(90 \%$ CI $)$ \\
\hline Ipatasertib & $\mathrm{AUC}_{0-\infty}(\mathrm{ng} * \mathrm{~h} / \mathrm{mL})$ & $545(496,598)$ \\
& $\mathrm{C}_{\max }(\mathrm{ng} / \mathrm{mL})$ & $226(183,280)$ \\
M1 (G-037720) & $\mathrm{AUC}_{0-72}(\mathrm{ng} * \mathrm{~h} / \mathrm{mL})$ & $31.6(26.7,37.6)$ \\
& $\mathrm{C}_{\max }(\mathrm{ng} / \mathrm{mL})$ & $8.9(7.6,10.5)$ \\
\hline
\end{tabular}

Itraconazole also appeared to impact the $t_{\max }$ of ipatasertib. The first detectable concentration and median $t_{\max }$ of ipatasertib was slightly delayed in the presence of itraconazole. This observation did not appear to be an artifact of sampling times or an anticipated effect of itraconazole-mediated CYP3A and P-gp inhibition. Given that the range of $t_{\text {max }}$ overlapped between the two treatment groups, it is likely that this observation was attributed to the high variability of ipatasertib PK. Also, for M1 (G-037720), the $t_{1 / 2}$ values should be interpreted with caution, as there were a limited number of quantifiable concentrations available to include in the estimation of the terminal rate constant. The apparent change in $\mathrm{V}_{\mathrm{z}} / \mathrm{F}$ in presence of itraconazole is likely due to reduced first pass effect, and therefore, a change in oral bioavailability ( $F)$.

Given the magnitude of the observed DDI, the coadministration of other strong CYP3A4 inhibitors such as posaconazole, clarithromycin, and grapefruit juice are being avoided in clinical studies with ipatasertib or used with a reduced dose of ipatasertib (https://www.fda.gov/drugs/drug-interactions-labeling /drug-development-and-drug-interactions-table-substratesinhibitors-and-inducers). In this study, a 100-mg single dose of ipatasertib was administered, which is lower than the intended therapeutic dose of $400 \mathrm{mg}$. At $400 \mathrm{mg}$, the magnitude of DDI is expected to be somewhat lower than that observed at $100 \mathrm{mg}$, as the competition for CYP3A4 is typically concentration-dependent. Physiologically based PK modeling is ongoing to predict the DDI at 200- and 400-mg dose of ipatasertib to provide guidance for use with weak and moderate inhibitors of CYP3A4.

The PK of itraconazole and its primary metabolite, 1hydroxyitraconazole, were also assessed in this study to ensure sufficient inhibitor concentrations were achieved. The $\mathrm{C}_{\max }$ and $\mathrm{t}_{\max }$ of itraconazole were comparable to that previously observed after administration of $200 \mathrm{mg}$ itraconazole oral solution under fasted conditions for 15 days at $1963 \mathrm{ng} /$ $\mathrm{mL}$ and 2.5 hours, respectively (Sporanox oral solution United States Prescribing Information). The $\mathrm{C}_{\max }$ of 1-hydroxyitraconazole observed on day 18 in this study was slightly lower than that previously reported; however, the geometric mean concentrations of itraconazole and 1-hydroxyitraconazole were well above the reported $\mathrm{IC}_{50}$ values [approximately $29 \mathrm{nM}$ (20.5 ng/mL) and $37 \mathrm{nM}(26.7 \mathrm{ng} / \mathrm{mL})$, respectively], indicating they provided sufficient inhibition of CYP3A4 in this study (Isoherranen et al., 2004).

Initial single or multiple ascending dose studies may often be desired to evaluate the effect of a drug on endogenous biomarkers. However, ipatasertib is in late stages of clinical 
TABLE 3

Summary of PK parameters of M1 (G-037720) after single oral dose of ipatasertib $100 \mathrm{mg}$ alone or coadministered with itraconazole $200 \mathrm{mg}$

All values are reported as geometric mean (geo\%CV); $t_{\max }$ is reported as median (range); $t_{1 / 2}$ is reported as mean (S.D.).

\begin{tabular}{lcc}
\hline M1 (G-037720) PK Parameters & Ipatasertib (100 mg) & Ipatasertib (100 mg) + Itraconazole (200 mg) \\
\hline & $N=15$ & $N=15$ \\
$\mathrm{AUC}_{0-\infty}(\mathrm{ng} * \mathrm{~h} / \mathrm{mL})$ & $233(35.7)^{\mathrm{a}}$ & $110(57.7)^{\mathrm{b}}$ \\
$\mathrm{AUC}_{0-72}(\mathrm{ng} / \mathrm{h} / \mathrm{mL})$ & $179(34.7)$ & $58.0(38.5)^{\mathrm{a}}$ \\
$\mathrm{C}_{\max }(\mathrm{ng} / \mathrm{mL})$ & $18.4(42.3)$ & $1.64(29.5)$ \\
$\mathrm{t}_{\max }(\mathrm{h})$ & $2.03(1.02,3.15)$ & $8.00(2.00,12.00)$ \\
$t_{1 / 2}(\mathrm{~h})$ & $47.6(14.0)$ & $36.1(12.7)^{\mathrm{a}}$ \\
$\mathrm{MR}_{\mathrm{amax}}$ & $0.452(50.2)$ & $0.0178(41.0)$ \\
$\mathrm{MR}_{\text {AUC }}$ & $0.787(19.8)$ & $0.0646(33.9)^{\mathrm{b}}$ \\
$\mathrm{MR}_{\mathrm{AUC} 0-72}$ & $0.728(20.1)$ & $0.0484(23.7)^{\mathrm{a}}$ \\
\hline
\end{tabular}

${ }^{\mathrm{a}} \mathrm{N}=14$.

${ }^{\mathrm{b}} \mathrm{N}=4$.

development with several patient studies ongoing in combination with other agents (Kim et al., 2017; de Bono et al., 2019). This study in healthy subjects was considered suitable to evaluate the in vivo effect of ipatasertib on OATP1B1/1B3, since ipatasertib was given as a single agent. In typical oncology clinical studies, patients are taking other comedications that can confound DDI study results. Therefore, it was important to have a study population with minimal confounder effects, as there was expected to be minimal or no effect of ipatasertib inhibition on OATP1B1/1B3 in vivo. Any confounding effects due to comedications could have made the results inconclusive. Based on in vitro predictions using the methods described in the FDA DDI guidance, at the clinically intended dose of $400 \mathrm{mg}$, and the observed $\mathrm{IC}_{50}$ values, the predicted AUC ratios (AUC in presence of inhibitor/AUC in absence of inhibitor), using the estimated maximum unbound liver inlet concentration $\left(\mathrm{I}_{\text {inlet,unbound }}\right)$ of ipatasertib and default values for
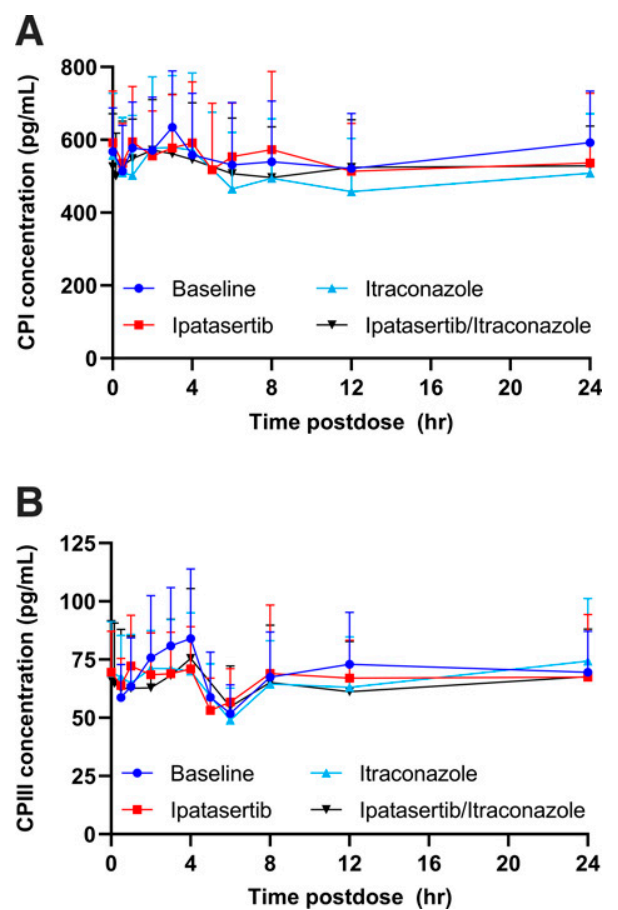

Fig. 5. Arithmetic mean (+S.D.) concentration-time profile of CPI (A) and CPIII (B) at baseline and after oral administration of ipatasertib $100 \mathrm{mg}$ alone, itraconazole $200 \mathrm{mg}$ alone, or coadministration of ipatasertib and itraconazole in healthy subjects $(N=15)$. fraction absorbed $(\mathrm{Fa})$, intestinal availability $(\mathrm{Fg})$ and absorption rate constant ( $\mathrm{Ka}$ ) (as worst case scenario), were calculated to be 1.25 and 1.19 for OATP1B1 and OATP1B3 inhibition, respectively. These are slightly higher than the FDA cutoff of 1.1 (FDA DDI guidance). However, when using a realistic Ka value estimated from population PK analysis of ipatasertib (data on file) to calculate the maximum unbound liver inlet concentration, these ratios were 1.08 and 1.06, respectively, for OATP1B1 and OATP1B3, less than the FDA cutoff of 1.1. Therefore, this CYP3A4 DDI study was leveraged to measure the changes in CPI and CPIII levels before and after administration of ipatasertib. As expected, after $100 \mathrm{mg}$ ipatasertib administration, no change was observed in CPI and CPIII exposures, which were surrogates for OATP1B1/ 1B3 activity.

In this present clinical DDI study, subjects received $100 \mathrm{mg}$ ipatasertib, which was lower than intended clinical dose of $400 \mathrm{mg}$ (Kim et al., 2017; de Bono et al., 2019). This dose was selected for the study to minimize unnecessary drug exposure in healthy subjects, especially in anticipation of a DDI. Consequently, the exposure of ipatasertib in presence of itraconazole was comparable to the exposure observed after administration of $400 \mathrm{mg}$ ipatasertib in patients. Therefore, CPI and CPIII were also evaluated in presence of both ipatasertib and itraconazole to assess the impact of ipatasertib exposures expected at intended therapeutic dose. There was no change in CPI and CPIII plasma levels even at higher ipatasertib exposures, indicating ipatasertib did not inhibit OATP1B1/1B3 in vivo at the clinically relevant exposures. After administration of ipatasertib alone, itraconazole alone, or coadministration of ipatasertib with itraconazole, $\mathrm{AUC}_{0 \text {-tz }}$ and $\mathrm{C}_{\max }$ of $\mathrm{CPI}$ and III were generally similar to the respective values at baseline (maximum decreases of $\leq 11 \%$ from baseline). Given that itraconazole also did not impact CPI and CPIII exposure, the conclusion that ipatasertib did not impact CPI and CPIII levels when coadministered with itraconazole was not confounded. Overall, OATP1B1/1B3 substrates can be coadministered with ipatasertib without a risk of DDI even at clinically relevant concentrations of ipatasertib.

To facilitate interpretation of CPI and CPIII data, an uptake experiment was prospectively conducted which demonstrated that neither CPI nor CPIII were substrates of P-gp. Itraconazole, a P-gp inhibitor, did not alter CPI and CPIII levels in our study, and this is consistent with an observed lack of P-gp involvement in CPI and CPIII transport (Fig. 3). This 
TABLE 4

Summary of parameters for CPI and CPIII at baseline and after administration of ipatasertib $100 \mathrm{mg}$ alone, Itraconazole $200 \mathrm{mg}$ alone, or coadministration of ipatasertib with Itraconazole

Data are expressed as Mean (S.D.).

\begin{tabular}{|c|c|c|c|c|c|}
\hline Analyte & Parameter & Baseline & $\begin{array}{l}\text { Ipatasertib } \\
(100 \mathrm{mg})\end{array}$ & $\begin{array}{l}\text { Itraconazole } \\
\quad(200 \mathrm{mg})\end{array}$ & $\begin{array}{c}\text { Ipatasertib }(100 \mathrm{mg})+ \\
\text { Itraconazole }(200 \mathrm{mg})\end{array}$ \\
\hline \multirow[b]{2}{*}{ CPI } & & $N=15$ & $N=15$ & $N=15$ & $N=15$ \\
\hline & $\mathrm{AUC}_{0-24}(\mathrm{pg} * \mathrm{~h} / \mathrm{mL})$ & $13300(3340)$ & $13000(3670)$ & $11900(3710)$ & $12500(2990)$ \\
\hline \multirow[t]{2}{*}{ CPIII } & AUC0-24 $(\mathrm{pg} * \mathrm{~h} / \mathrm{mL})$ & $1670(443)$ & $1600(446)$ & $1580(492)$ & $1530(499)$ \\
\hline & $\mathrm{C}_{\max }(\mathrm{pg} / \mathrm{mL})$ & $89.8(28.9)$ & $85.7(26.3)$ & $82.4(23.7)$ & $82.0(28.3)$ \\
\hline
\end{tabular}

observation was in line with previously reported in vitro data; CPI and CPIII have been reported as being substrates of OATP1B1/3 and the multidrug resistance-associated protein (MRP) 2 and MRP3. CPI and CPIII were not substrates of other uptake or efflux transporters including BCRP, the bile salt export pump, P-gp, the multidrug and toxin extrusion proteins $1 / 2 \mathrm{~K}, \mathrm{MRP} 4$, the OAT $1 / 3$, the organic cation transporter $1 / 2$, and the sodium taurocholate cotransporting polypeptide (Kunze et al., 2018).

The observation that CPI and CPIII levels were not altered in a clinical setting by the presence of itraconazole, which is an inhibitor or CYP3A4, P-gp, and BCRP, adds to the scientific knowledge in the field of transporter-mediated DDI. Itraconazole has been shown to increase the concentration of OATP1B1/1B3 substrates such as rosuvastatin and pravastatin in previous studies (Vaidyanathan et al., 2016). However, the lack of effect on CPI and CPIII in our study suggests inhibition of other transporters such as P-gp and/or BCRP by itraconazole and/or 1-hydroxyitraconazole may be playing a role in the DDI studies with statins. If a new molecule is an inhibitor of CYP3A4 and multiple transporters such as P-gp, BCRP, and OATP1B1/1B3, CPI and CPIII offer an appropriate option to evaluate an exclusive effect on OATP1B1/1B3 as compared with probe substrates such as atorvastatin, rosuvastatin, or simvastatin, which are substrates of these other enzymes or transporters. This study demonstrates, in a clinical setting, that CPI and CPIII exposures are not affected by itraconazole, a CYP3A4, P-gp, and BCRP inhibitor. These results make interpretation of itraconazole DDI studies simpler, when the victim molecules are substrates of multiple transporters, such as fexofenadine (Shimizu et al., 2006). This type of study design also offers the flexibility to evaluate CPI and CPIII at a higher concentration of the inhibitor drug, which may be needed for studying complex DDI or DDI in special populations, scenarios in which

\section{TABLE 5}

Statistical analysis of the effect of ipatasertib on PK parameters of Coproporphyrins I and III

Geometric mean ratio (GMR) is the ratio of the least squares means from ANOVA for natural log-transformed $\mathrm{AUC}_{0-24}$ or $\mathrm{C}_{\text {max }}$ (expressed as a percent), natural log-transformed back to the linear scale; Test/Reference where test $=$ ipatasertib $100 \mathrm{mg}$ alone and reference $=$ Baseline

\begin{tabular}{lcc}
\hline Analyte & \multicolumn{1}{c}{ Parameter } & GMR $(90 \%$ CI $)$ \\
\hline \multirow{2}{*}{ Coproporphyrin I } & $\mathrm{AUC}_{0-24}(\mathrm{pg} * \mathrm{~h} / \mathrm{mL})$ & $97.4(94.0,100.8)$ \\
& $\mathrm{C}_{\max }(\mathrm{pg} / \mathrm{mL})$ & $96.7(88.4,105.9)$ \\
Coproporphyrin III & $\mathrm{AUC}_{0-24}(\mathrm{pg} * \mathrm{~h} / \mathrm{mL})$ & $95.9(89.6,102.8)$ \\
& $\mathrm{C}_{\max }(\mathrm{pg} / \mathrm{mL})$ & $95.8(86.4,106.2)$ \\
\hline
\end{tabular}

higher drug exposures may occur. Itraconazole can boost perpetrator concentrations which may aid CPI and CPIII evaluation at a higher inhibitor concentration without any interference.

Overall, using this approach of evaluating two DDIs in one study has provided evidence that ipatasertib is a substrate of CYP3A4 in vivo and therefore a victim of DDI with strong CYP3A4 inhibitors, and that ipatasertib does not inhibit OATP1B1/1B3 in vivo. As polypharmacy is not uncommon in patients with cancer, this study has provided critical information to ensure safe use of ipatasertib in patients and provides DDI risk mitigation strategies for ongoing clinical studies and future clinical use of ipatasertib. The study also offers new insights by demonstrating lack of CPI/CPIII interaction with itraconazole.

\section{Acknowledgments}

We thank the research team at Covance Inc. for conducting this study.

\section{Authorship Contributions}

Participated in research design: Sane, Liederer, Malhi, Musib. Conducted experiments: Sane, Cho, Hanover, Plise, Wong.

Contributed new reagents or analytic tools: Sane, Plise.

Performed data analysis: Malhi, Liederer, Wong.

Wrote or contributed to the writing of the manuscript: Sane, Cheung, Liederer, Malhi, Plise.

\section{References}

Blake JF, Xu R, Bencsik JR, Xiao D, Kallan NC, Schlachter S, Mitchell IS, Spencer KL, Banka AL, Wallace EM, et al. (2012) Discovery and preclinical pharmacology of a selective ATP-competitive Akt inhibitor (GDC-0068) for the treatment of human tumors. J Med Chem 55:8110-8127.

de Bono JS, De Giorgi U, Rodrigues DN, Massard C, Bracarda S, Font A, Arranz Arija JA, Shih KC, Radavoi GD, Xu N, et al. (2019) Randomized phase ii study evaluating Akt blockade with ipatasertib, in combination with abiraterone, in patients with metastatic prostate cancer with and without PTEN loss. Clin Cancer Res 25:928-936.

Herédi-Szabó K, Palm JE, Andersson TB, Pál Á, Méhn D, Fekete Z, Beéry E, Jakab KT, Jani M, and Krajcsi P (2013) A P-gp vesicular transport inhibition assay - optimization and validation for drug-drug interaction testing. Eur J Pharm Sci 49:773-781.

Isakoff SJ, Tabernero J, Molife LR, Soria JC, Cervantes A, Vogelzang NJ, Patel MR, Hussain M, Baron A, Argilés G, et al. (2020) Antitumor activity of ipatasertib combined with chemotherapy: results from a phase Ib study in solid tumors. Ann Oncol 31(5):626-633.

Isoherranen N, Kunze KL, Allen KE, Nelson WL, and Thummel KE, (2004) Role of itraconazole metabolites in CYP3A4 inhibition. Drug Metab Dispos 32:1121-1131.

Kim SB, Dent R, Im SA, Espié M, Blau S, Tan AR, Isakoff SJ, Oliveira M, Saura C, Wongchenko MJ, et al.; LOTUS investigators (2017) Ipatasertib plus paclitaxel versus placebo plus paclitaxel as first-line therapy for metastatic triple-negative breast cancer (LOTUS): a multicentre, randomised, double-blind, placebo-controlled, phase 2 trial. Lancet Oncol 18:1360-1372.

Kunze A, Ediage EN, Dillen L, Monshouwer M, and Snoeys J (2018) Clinical investigation of coproporphyrins as sensitive biomarkers to predict mild to strong OATP1B-mediated drug-drug interactions. Clin Pharmacokinet 57:1559-1570. 
Lai Y, Mandlekar S, Shen H, Holenarsipur VK, Langish R, Rajanna P, Murugesan S, Gaud N, Selvam S, Date O, et al. (2016) Coproporphyrins in plasma and urine can be appropriate clinical biomarkers to recapitulate drug-drug interactions mediated by organic anion transporting polypeptide inhibition. J Pharmacol Exp Ther 358:397-404

Lin J, Sampath D, Nannini MA, Lee BB, Degtyarev M, Oeh J, Savage H, Guan Z, Hong R, Kassees R, et al. (2013) Targeting activated Akt with GDC-0068, a novel selective Akt inhibitor that is efficacious in multiple tumor models. Clin Cancer Res 19:1760-1772.

Liu L, Cheeti S, Yoshida K, Choo E, Chen E, Chen B, Gates M, Singel S, Morley R, Ware J, et al. (2018) Effect of OATP1B1/1B3 inhibitor GDC-0810 on the pharmacokinetics of pravastatin and coproporphyrin I/III in healthy female subjects. J Clin Pharmacol 58:1427-1435.

Prueksaritanont T, Tatosian DA, Chu X, Railkar R, Evers R, Chavez-Eng C, Lutz R, Zeng W, Yabut J, Chan GH, et al. (2017) Validation of a microdose probe drug cocktail for clinical drug interaction assessments for drug transporters and CYP3A. Clin Pharmacol Ther 101:519-530 10.1002/cpt.525.

Sane R, Cheung KWK, Kovács P, Farasyn T, Li R, Bui A, Musib L, Kis E, Plise E, and Gáborik Z (2020) Calibrating the in vitro-in vivo correlation for OATP- mediated drug-drug interactions with rosuvastatin using static and PBPK models. Drug Metab Dispos 48:1264-1270.

Shimizu M, Uno T, Sugawara K, and Tateishi T (2006) Effects of single and multiple doses of itraconazole on the pharmacokinetics of fexofenadine, a substrate of P-glycoprotein. Br J Clin Pharmacol 62:372-376.

Vaidyanathan J, Yoshida K, Arya V, and Zhang L (2016) Comparing various in vitro prediction criteria to assess the potential of a new molecular entity to inhibit organic anion transporting polypeptide 1B1. J Clin Pharmacol 56 (Suppl 7):S59-S72.

Venkatakrishnan K, von Moltke LL, and Greenblatt DJ (2000) Effects of the antifungal agents on oxidative drug metabolism: clinical relevance. Clin Pharmacokinet 38:111-180.

Yan Y, Serra V, Prudkin L, Scaltriti M, Murli S, Rodríguez O, Guzman M, Sampath D, Nannini M, Xiao Y, et al. (2013) Evaluation and clinical analyses of downstream targets of the Akt inhibitor GDC-0068. Clin Cancer Res 19:6976-6986.

Address correspondence to: Dr. Rucha Sane, Department of Clinical Pharmacology Genentech, 1 DNA Way, South San Francisco, CA 94080. E-mail: saner@gene.com 